\title{
A fast correction for traction-free surface of elastic quarter-space
}

\author{
R. Guilbault \\ Department of Mechanical Engineering, \\ École de technologie supérieure, Canada
}

\begin{abstract}
Applying the Hertz theory to some non-Hertzian contact problems can produce acceptable results. Nevertheless, including the influence of free surfaces requires numerical methods, many of which are based on the Boussinesq-Cerruti solution. This paper presents a new approach which is better capable of releasing quarter-space free surfaces from shear and normal internal stresses without any increase in calculation times. The mirrored pressure for shear correction is multiplied by a correction factor $(\psi)$, which accounts for the normal load. The expression $\psi$ is derived from the Hetényi correction process, and the resulting displacements show an enhanced correspondence with validation FEM models; with an imposed fluctuating pressure, the maximum edge displacement error was $-21,90 \%$ for a shear load correction (Poisson coefficient $v=0,3$ ), and introducing the $\psi$ factor reduced the deviation to $-9,55 \%$, while for $\mu$ of 0,15 , the maximum error was $-11,30 \%$, which was reduced to $+0,60 \%$ with the $\psi$ factor.
\end{abstract}

Keywords: quarter-space, quarter-plane, free surface, contact model, Boussinesq-Cerruti solution, normal load.

\section{Introduction and literature survey}

The Hertz theory can be applied to some non-Hertzian contact problems, and lead to acceptable results even when basic hypotheses are not fully respected. Such applications remain but approximated treatments, since the Hertz theory does not include the influence of free surfaces. In reality, including the influence of free surfaces requires numerical methods, the best-known being the finite element method (FEM). However, the computation time of FEM contact models can still have detrimental consequences. Fortunately, other accurate numerical 
approaches exist, which are potentially more efficient in term of computation time.

Many 2D and 3D methods are based on the Boussinesq-Cerruti solution for point traction acting on elastic half-space (Hetényi [1, 2], Keer et al [3], Hartnett [4], Poon and Sayles [5], Li and Berger [6], and Chen and Wang [7]), where influence functions link surface tractions and displacements (Love [8], Li and Berger [6]). However, when used without correction in contact representations, such as in the Hartnett model (Hartnett [4]) these influence functions generate incorrect pressure increases in vicinity of free surfaces of real bodies. On the other hand, in contact problems with no free-surface effects, the Hartnett model produces very rapid and precise solutions. For this reason, many authors (Poon and Sayles [5], Chen and Wang [7]) have employed analogous modelling techniques for a variety of no-free-surface contact problems, and obtained accurate results.

The internal shear and normal stresses introduced by the Boussinesq-Cerrutibased methods on traction-free surfaces were already studied and solved by previous authors (Hetényi [1, 2], Keer et al [3]), and the free surface internal stresses are illustrated in fig. 1. The correction methods they proposed are exact and lead to a complete release of free surfaces. The technique requires repeated superpositions of the solution for the elastic half-space and then, significant computation efforts. Therefore, other authors (de Mul et al [9]) have observed that the shear load $(\tau)$ greatly influences the loaded surface displacement, while the normal load $(\sigma)$ only plays a minor role. Consequently, as suggested by the Hetényi solution, a partial correction could be obtained when the pressure distribution is mirrored with respect to the free surfaces.

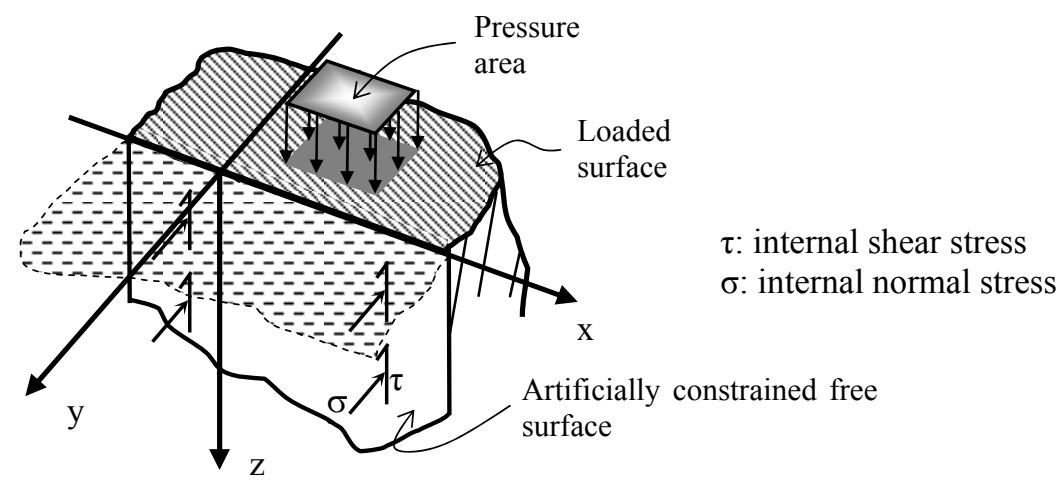

Figure 1: $\quad$ Half-space free surface internal stresses.

Since a partial correction does not noticeably increase the calculation time, yet offers visible gains in precision, this method has been successfully employed in machine element modelling (Guilbault et al [10]). However, in sensitive 
applications, the remaining $\sigma$ could generate inadequate displacement calculation close to limiting edges of finite dimension bodies.

This paper presents a new approach which is better capable of releasing quarter-space free surfaces from $\tau$ and $\sigma$ stresses without requiring increased calculation times as compared to the partial correction. The mirrored pressure for the $\tau$ correction is multiplied by a correction factor $(\psi)$, which introduces an overcorrection to account for $\sigma$. The expression $\psi$ is derived from the Hetényi correction process. Even though the proposed numerical approach is still an approximated correction, the resulting displacements show a good correspondence with FEM validation models.

\section{Internal stresses}

If a constant pressure $\left(\mathrm{p}_{\mathrm{ij}}\right)$ is applied over a rectangular area $(2 \mathrm{a} X 2 \mathrm{~b})$ of an elastic half-space, the normal displacement $\left(\delta_{\mathrm{kc}}\right)$ of any surface point $k c$ is calculated with eqns (1) and (2) (Love [8]). These equations are illustrated in fig. 2.

$$
\begin{aligned}
& f_{k c, i j}=\left\{\frac{1-v^{2}}{\pi E}\right\}\left\{\begin{array}{l}
\left(\eta_{s}+b\right) \ln \left[\frac{\left(\xi_{s}+a\right)+\left\{\left(\eta_{s}+b\right)^{2}+\left(\xi_{s}+a\right)^{2}\right\}^{1 / 2}}{\left(\xi_{s}-a\right)+\left\{\left(\eta_{s}+b\right)^{2}+\left(\xi_{s}-a\right)^{2}\right\}^{1 / 2}}\right] \\
{\left[\begin{array}{l}
\left(\eta_{s}+b\right)+\left\{\left(\eta_{s}+b\right)^{2}+\left(\xi_{s}+a\right)^{2}\right\}^{1 / 2} \\
{\left[\left(\eta_{s}-b\right)+\left\{\left(\eta_{s}-b\right)^{2}+\left(\xi_{s}+a\right)^{2}\right\}^{1 / 2}\right.}
\end{array}\right\}} \\
\left(\xi_{s}-a\right) \ln \left[\frac{\left(\eta_{s}-b\right)+\left\{\left(\eta_{s}-b\right)^{2}+\left(\xi_{s}-a\right)^{2}\right\}^{1 / 2}}{\left(\eta_{s}+b\right)+\left\{\left(\eta_{s}+b\right)^{2}+\left(\xi_{s}-a\right)^{2}\right\}^{1 / 2}}\right] \\
{\left[\eta_{s}-b\right) \ln \left[\frac{\left(\xi_{s}-a\right)+\left\{\left(\eta_{s}-b\right)^{2}+\left(\xi_{s}-a\right)^{2}\right\}^{1 / 2}}{\left(\xi_{s}+a\right)+\left\{\left(\eta_{s}-b\right)^{2}+\left(\xi_{s}+a\right)^{2}\right\}^{1 / 2}}\right]}
\end{array}\right\} \\
& \delta_{k c}=p_{i j} \times f_{k c, i j}
\end{aligned}
$$

where $\xi$ and $\eta$ are local coordinates, $\xi_{\mathrm{s}}=\xi_{\mathrm{kc}}-\xi_{\mathrm{ij}}$ and $\eta_{\mathrm{s}}=\eta_{\mathrm{kc}}-\eta_{\mathrm{ij}}, \nu=$ Poisson coefficient and $\mathrm{E}=$ Young's modulus.

Figure 2 also illustrates the $\tau$ elimination by the mirroring operation. In this representation, four correction mirrors are required because of the presence of two traction-free surfaces.

Hetényi presented a correction method for both the quarter-plane (Hetényi [1]) and the quarter-space problems (Hetényi [2]). After the mirror $\tau$ correction process, the $\sigma$ distribution, which is actually doubled by the mirror 


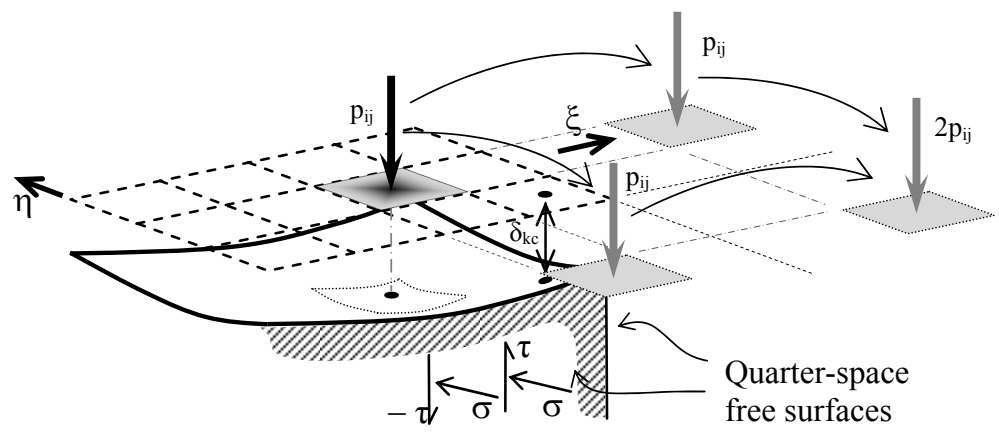

Figure 2: $\quad$ Pressure cells and mirror correction.

pressure application, is regarded as a distributed normal loading, and counterbalanced by a normal force distribution of the opposite sign.

The free surfaces must then be meshed, and the correction force distributions integrated into the problem by means of eqns (1) and (2). Since the correction forces generate new internal shear and normal stresses on the boundary, the mirroring process and normal forces elimination need to be repeated. The iterative procedure results in a complete correction of the free line or surface of the quarter-plane or space.

When considering the half-plane problem subjected to two concentrated forces $P$ (fig. 3 ), the stress components at a given point $(x, z)$ are given by eqns (3)

$$
\begin{aligned}
& \sigma_{x}=-\frac{2 P}{\pi}\left\{\frac{z(x+a)^{2}}{\left(z^{2}+(x+a)^{2}\right)^{2}}+\frac{z(x-a)^{2}}{\left(z^{2}+(x-a)^{2}\right)^{2}}\right\} \\
& \sigma_{z}=-\frac{2 P}{\pi}\left\{\frac{z^{3}}{\left(z^{2}+(x+a)^{2}\right)^{2}}+\frac{z^{3}}{\left(z^{2}+(x-a)^{2}\right)^{2}}\right\} \\
& \tau_{x z}=-\frac{2 P}{\pi}\left\{\frac{z^{2}(x+a)}{\left(z^{2}+(x+a)^{2}\right)^{2}}+\frac{z^{2}(x-a)}{\left(z^{2}+(x-a)^{2}\right)^{2}}\right\}
\end{aligned}
$$

At position $x=0$ eqns (3) become

$$
\begin{gathered}
\sigma_{x}=-\frac{4 P}{\pi}\left\{\frac{z(x+a)^{2}}{\left(z^{2}+(x+a)^{2}\right)^{2}}\right\} \\
\sigma_{z}=-\frac{4 P}{\pi}\left\{\frac{z^{3}}{\left(z^{2}+(x+a)^{2}\right)^{2}}\right\} \\
\tau_{x z}=0
\end{gathered}
$$




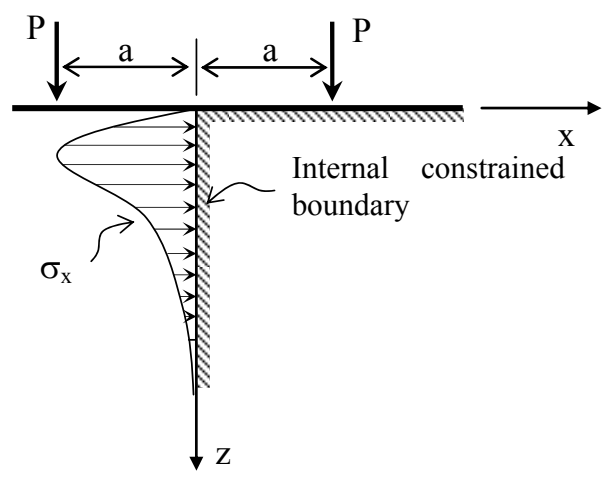

Figure 3: Half-plane with concentrated forces.

Integrating eqn (4a) with respect to $z$ gives the total lateral force constraining the $z$-axis (eqn (5)).

$$
\int_{0}^{\infty} \sigma_{x} d z=-2 P \frac{1}{\pi}
$$

Eqn (5) indicates that the resulting force amplitude is equal to the total applied load divided by $\pi$. Consequently, the elimination of this surface loading will, in turn, generate an additional normal load $(2 / \pi)^{2} \mathrm{P}$ on the initial boundary, leading to geometric convergent series. Moreover, the line of action of the lateral force is determined as follows:

$$
\frac{\int_{0}^{\infty} z \sigma_{x} d z}{\int_{0}^{\infty} \sigma_{x} d z}=\frac{\pi}{2} a
$$

Eqn (6) shows that while the amplitude of the lateral force decreases, its position shifts away from the considered boundary. While the author did indeed establish the convergence of the process in a slightly different manner, this clearly illustrates the convergence of the Hetényi iterative process.

Hetényi ultimately formulated the total normal load acting on the initial halfplane boundary $(z=0)$ and on the boundary $(x=0)$ from eqns (7) and (8), respectively, where $\left[\sigma_{\mathrm{z}}\right]_{\mathrm{z}=0}$ and $\left[\sigma_{\mathrm{x}}\right]_{\mathrm{x}=0}$ are the cumulative stress distributions generated during the successive corrections. 
42 Tribology and Design

$$
\begin{aligned}
& C_{z}=\int_{0}^{\infty}\left[\sigma_{z}\right]_{z=0} d x=\frac{P}{a}\left(\frac{4}{\pi^{2}-4}\right) \\
& C_{x}=\int_{0}^{\infty}\left[\sigma_{x}\right]_{x=0} d z=\frac{P}{a}\left(\frac{2 \pi}{\pi^{2}-4}\right)
\end{aligned}
$$

From eqns (7) and (8), Hetényi clearly demonstrated the complete elimination of the initial lateral load established with eqn (5). The inclusion of the third dimension in eqn (5) would still give the same lateral load amplitude $(2 \mathrm{P} / \pi)$. Figure 4 illustrates the quarter-plane free boundary correction operation.

(A) Initial state

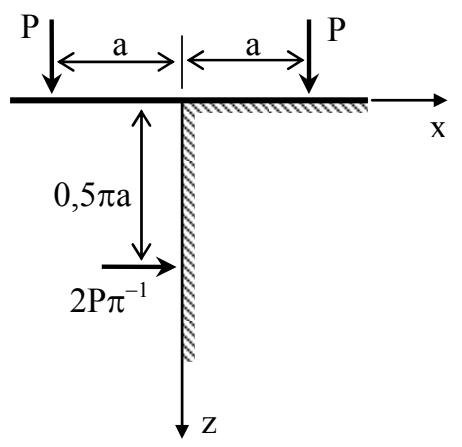

Figure 4: Quarter-plane states.

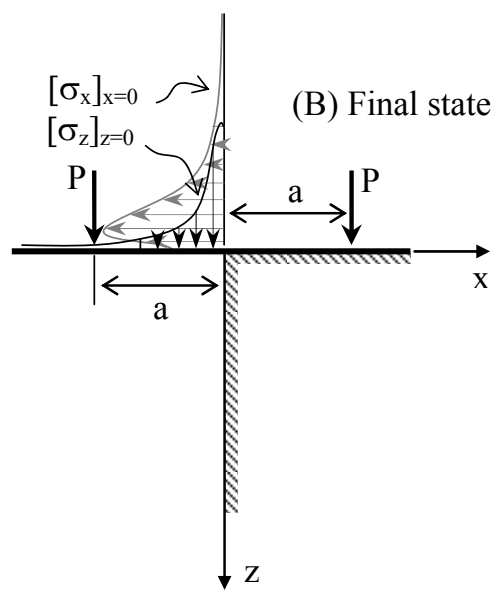

Since the objective of the present study is not to reproduce the Hetényi iterative process, which could be cumbersome, but rather, to compensate for the influence of the internal normal stress on the $\delta_{\mathrm{kc}}$ amplitude, the following development of the factor $\psi$ does not include the stress distributions, but rather, the corresponding concentrated forces.

\section{Surface displacement}

The displacements at any points in a solid subjected to a concentrated normal force $P$ in the $z$ direction are given by eqns (9).

$$
\begin{aligned}
& u_{x}(P)=\frac{P(1+v)}{2 \pi E}\left\{\frac{x z}{\rho^{3}}-(1-2 v) \frac{x}{\rho(\rho+z)}\right\} \\
& u_{y}(P)=\frac{P(1+v)}{2 \pi E}\left\{\frac{y z}{\rho^{3}}-(1-2 v) \frac{y}{\rho(\rho+z)}\right\}
\end{aligned}
$$




$$
u_{z}(P)=\frac{P(1+v)}{2 \pi E}\left\{\frac{z^{2}}{\rho^{3}}+\frac{2(1-v)}{\rho}\right\}
$$

where $\rho=\sqrt{x^{2}+y^{2}+z^{2}}$.

If the quarter-space is reduced to a quarter-plane, fig. 5 illustrates the load conditions of the released half-plane (fig. (4(B))).

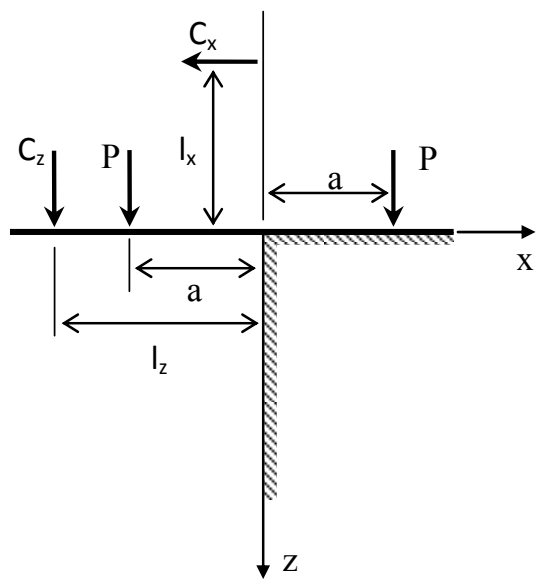

Figure 5: Quarter-plane conditions.

To quantify the influence of the correction forces $\left(C_{x}\right.$ and $\left.C_{z}\right)$ shown in fig. 5 on the $z$ displacement at the point of application of $P$, eqns (9) can be rewritten as:

$$
\begin{gathered}
u_{z}\left(C_{x}\right)=\delta\left(C_{x}\right)=\frac{C_{x}(1+v)}{2 \pi E}\left\{\frac{a l_{x}}{\rho^{3}}-(1-2 v) \frac{l_{x}}{\rho(\rho+a)}\right\} \\
u_{z}\left(C_{z}\right)=\delta\left(C_{z}\right)=\frac{C_{z}(1+v)}{2 \pi E}\left\{\frac{2(1-v)}{l_{z}+a}\right\} \\
u_{z}(P)=\delta(P)=\frac{P(1+v)}{2 \pi E}\left\{\frac{2(1-v)}{2 a}\right\} \quad \text { (mirror force) }
\end{gathered}
$$

where $\rho=\sqrt{l_{z}^{2}+a^{2}}, l_{x}<0$ and $C_{x}<0$.

The cumulated influence of these corrective loads could be simulated by the mirroring process with an artificial overcorrection $\mathrm{P}^{*}$ (eqn (11)). 
44 Tribology and Design

$$
\delta\left(P^{*}\right)=\delta(P)+\delta\left(C_{x}\right)+\delta\left(C_{z}\right)
$$

This equation states that the elastic inherent rigidity of the half-space could be compensated through the addition of a displacement $\delta$ produced by a mirror corrective pressure $\mathrm{P}^{*}$. The $\mathrm{P}^{*}$ amplitude must then be calculated with respect to the free surface in a given direction, allowing the direct transposition of the quarter-plane solution to the quarter-space problem. Replacing the real cumulative corrective load distributions with the equivalent forces $\left(C_{x}\right.$ and $\left.C_{z}\right)$ for speed gain will influence the final precision of the correction. That however, is an acceptable trade-off.

A single level $(n=1)$ of correction produces: $C_{x}=-(2 / \pi) P, l_{x}=-(\pi / 2) a$ and $C_{z}=(2 / \pi)^{2} P, l_{z}=(\pi / 2)^{2} a$. The corresponding correction factor $\psi$ calculated form eqn (11) is then:

$$
\psi=\frac{P^{*}}{P}=\left\{1+\frac{2^{5}}{\pi^{2}\left(\pi^{2}+2^{2}\right)}+\frac{1}{(1-v)}\left[\frac{1}{\left(\left(\frac{\pi}{2}\right)^{2}+1\right)^{\frac{3}{2}}}-\frac{(1-2 v)}{\left(\left(\frac{\pi}{2}\right)^{2}+1\right)^{\frac{1}{2}}\left(\left(\left(\frac{\pi}{2}\right)^{2}+1\right)^{\frac{1}{2}}+1\right)}\right]\right\}
$$

This first stage correction reduces the initial lateral load $(2 / \pi) \mathrm{P}$ to $(2 / \pi)^{3} \mathrm{P}$ by the equivalent of $59,5 \%$, whereas an $n \rightarrow \infty$ level of successive corrections theoretically lead to a complete release (eqn (13)). Eqn (13) is also a convergent series.

$$
\psi=\frac{P^{*}}{P}=\left\{\begin{array}{l}
1+\sum_{n=1}^{\infty} \frac{2^{4 n+1}}{\pi^{2 n}\left(\pi^{2 n}+2^{2 n}\right)}+ \\
\frac{1}{(1-v)}\left[\begin{array}{l}
\sum_{n=1}^{\infty} \frac{1}{\left(\left(\frac{\pi}{2}\right)^{2(2 n-1)}+1\right)^{\frac{3}{2}}} \\
\left.-(1-2 v) \sum_{n=1}^{\infty} \frac{1}{\left(\left(\frac{\pi}{2}\right)^{2(2 n-1)}+1\right)^{\frac{1}{2}}\left(\left(\left(\frac{\pi}{2}\right)^{2(2 n-1)}+1\right)^{\frac{1}{2}}+1\right.}\right)
\end{array}\right]
\end{array}\right\}
$$

Eqn (13) could be approximated by the following formulation. 


$$
\psi=\frac{P^{*}}{P}=1,29+\frac{1}{(1-v)}[0,17-0,25(1-2 v)]
$$

\section{Validation}

Eqn (14) is established to be integrated in a non-Hertzian contact model based on eqns (1) and (2). However, since the influence of the inherent normal stress can be minor in contact modeling, and consequently, veiled by the global precision. The validation models are built with predefined constant pressures applied over known areas, and three Poisson coefficient values $v$ are considered $(0,15 ; 0,3$ and $0,45)$. Figure 6 presents the tested form. The different cases are compared with FEM models, and to allow an isolation of the surface movement, the load is selfequilibrated.

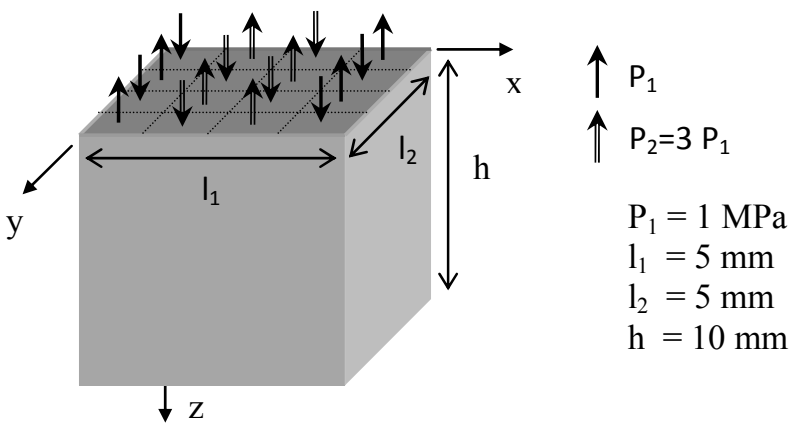

Figure 6: $\quad$ Test form.

The FEM models were meshed with parabolic tetrahedral elements of side length $0,125 \mathrm{~mm}$ through a $2,5 \mathrm{~mm}$ thickness ( $z$ direction). The remaining portion of the volume was meshed with gradual increasing element length (up to $1 \mathrm{~mm}$ ). The form was also treated with constant pressure square areas (side length $=0,0625 \mathrm{~mm}$ ), and the surface displacement was calculated with eqns (1) and (2), and corrected for internal stresses ( $\tau$ and $\sigma$ ) through the mirroring process combined with the overcorrection factor $\psi$.

For the sake of clarity, 2D charts are preferred. Figures 7, 8 and 9 thus present the displacement values along the edges of the loaded surface ( $x$ and $y=0$ ). Displacement values for a shear correction alone $(\psi=1)$ are also added.

A visual inspection of the charts reveals a general enhanced correspondence between the overcorrected results and the FEM evaluations, as compared to the shear correction alone $(\psi=1)$. The figures also indicate that slight differences remain, and increase with $v$. Figure 10 draws the maximum error curves for edges $y=0$ and $x=0$, respectively. These figures reveal that the edge displacement error (compared to FEM models) has an almost linear relation with the $v$ value. 

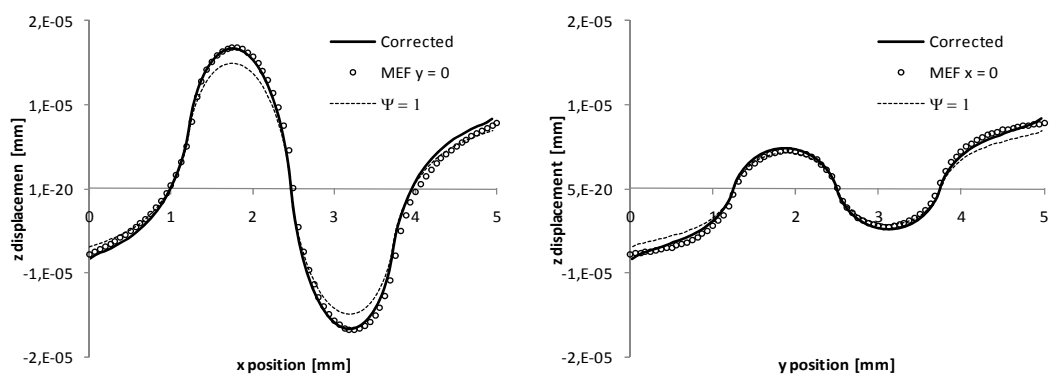

Figure 7: $\quad$ Edge displacement $(v=0,15)$.
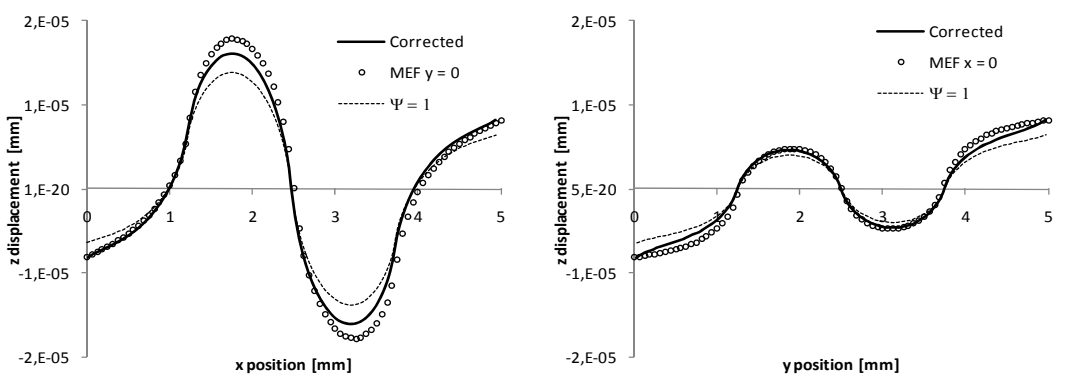

Figure 8: $\quad$ Edge displacement $(v=0,30)$.
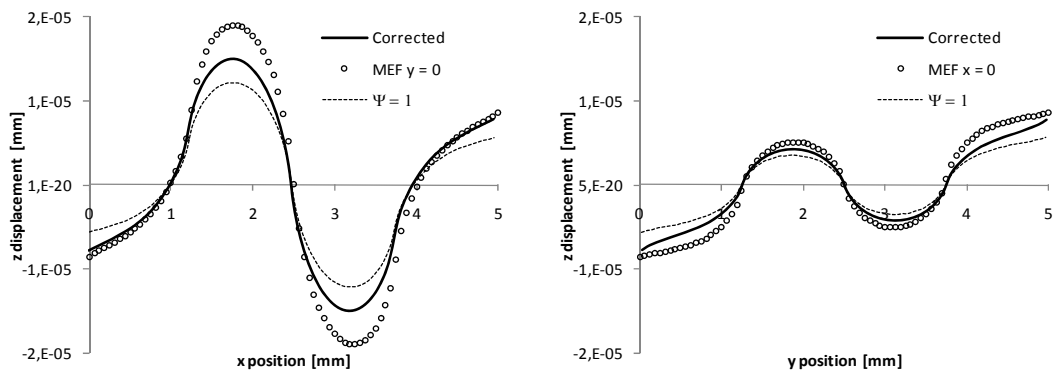

Figure 9: $\quad$ Edge displacement $(v=0,45)$.

The maximum edge displacement error (along $y=0$ edge) is $-11,3 \%$ for a shear stress correction $(\psi=1)$ when $v=0,15$, and introducing the $\psi$ factor reduces the deviation to $0,60 \%$. When $v=0,30$ and 0,45 , the maximum error amplitudes are $-21,9 \%$ and $-36,3 \%$ respectively, and are reduced to $-9,55 \%$ and $-21,1 \%$ with the $\psi$ factor. This indicates that the $\psi$ effectiveness varies with $v$; for edge $y=0$, the error reductions were $94,7 \%$ when $v=0,15,56,4 \%$ when $v=0,30$ and $42,0 \%$ when $v=0,45$. This persistent difference is attributable to the concentrated equivalent forces $C_{x}$ and $C_{z}$ used in place of the real distributions. 


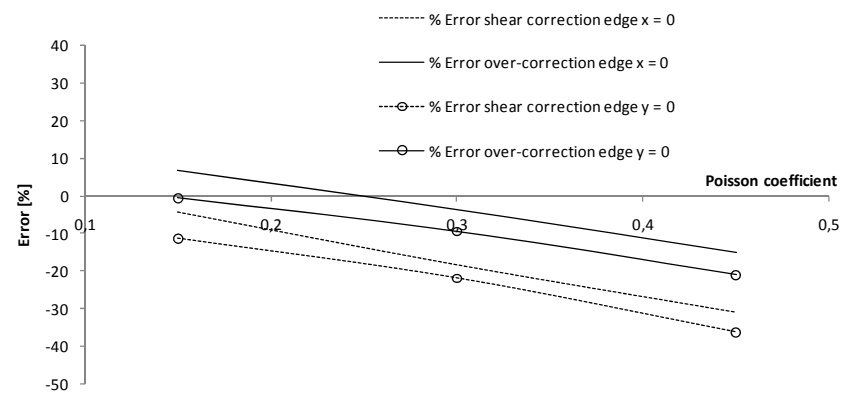

Figure 10: Maximum error curve along edge.

\section{Conclusion}

The Boussinesq and Cerruti non-Hertzian contact models offer precision and rapid solutions. However, the underlying half-space formulation introduces shear and normal stress distributions on traction-free surfaces. This paper presented the development of an overcorrection factor $\psi$ accounting for the normal stress in the mirroring process. Compared to FEM models, it has been shown that the introduction of the $\psi$ factor better simulates the elimination of the shear and normal stress influence on the displacement, and since no additional operations are necessary, the mirroring calculation times are not affected. The results reveal that the use of concentrated forces in place of real stress distributions causes deviations proportional to the Poisson coefficient of the material. When $v=0,3$, the presence of $\psi$ reduced the error on the edge from $-21,9 \%$ for shear correction to only $-9,55 \%$, representing a $56,4 \%$ error reduction.

\section{References}

[1] Hetényi, M., A method of solution for the elastic quarter-plane. J. of Appl. Mech., 82 (2), pp. 289-296, 1960.

[2] Hetényi, M., A general solution for the elastic quarter space. J. of Appl. Mech., 37(1), pp. 70-76, 1970.

[3] Keer, L.M., Lee, J.C. \& Mura, T., Hetényi’s elastic Quarter space problem revisited. Int. J. Solids Structures, 19(6), pp. 497-508, 1983.

[4] Hartnett, M. J., A general numerical solution for elastic body contact problems. Solid contact and lubrication, eds H. S. Cheng \& L. M. Keer AMD ASME, 39, pp. 51-66, 1980.

[5] Poon, C. Y. \& Sayles, R. S., Numerical Contact model of a smooth ball on an anisotropic rough surface. ASME J. Tribol., 116(2), pp. 194-201, 1994.

[6] Li J. \& Berger E.J., A Boussinesq-Cerruti solution set for constant and linear distribution of normal and tangential load over a triangular area. Journal of elasticity, 63(2), pp. 137-151, 2001. 
48 Tribology and Design

[7] Chen W. W. \& Wang, J. Q., A numerical static friction model for spherical contacts of rough surfaces, influence of load, material and roughness. ASME J. Tribol., 131(2), 0214028 pages, 2009.

[8] Love, A. E. H., The stress produced in a semi-infinite solid by pressure on part of the boundary. Phil. Trans. Royal Society of London, Series A, vol. 228, pp. 377-420, 1929.

[9] de Mul J. M., Kalker, J. J. \& Fredriksson, B., The contact between arbitrarily curved bodies of finite dimensions. ASME J. Tribol., 108, pp. 140-148, 1986.

[10] Guilbault, R., Gosselin, C., Cloutier, L., Express model for load sharing and stress analysis in helical gears. ASME J. Mech. Des., 127(6), pp. 11611172, 2005. 